\title{
A Modified 2D Multiresolution Hybrid Algorithm for Ultrasound Strain Imaging
}

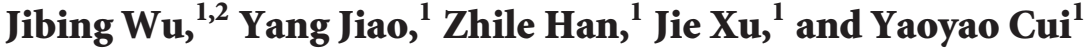 \\ ${ }^{1}$ Suzhou Institute of Biomedical Engineering and Technology, Chinese Academy of Sciences, Suzhou, Jiangsu 215163, China \\ ${ }^{2}$ University of Chinese Academy of Sciences, Beijing 100049, China \\ Correspondence should be addressed to Yaoyao Cui; cuiyy@sibet.ac.cn
}

Received 25 July 2017; Revised 29 October 2017; Accepted 15 November 2017; Published 20 December 2017

Academic Editor: Yongjin Zhou

Copyright (c) 2017 Jibing Wu et al. This is an open access article distributed under the Creative Commons Attribution License, which permits unrestricted use, distribution, and reproduction in any medium, provided the original work is properly cited.

\begin{abstract}
Ultrasound elastography is an imaging modality to evaluate elastic properties of soft tissue. Recently, 1D quasi-static elastography method has been commercialized by some companies. However, its performance is still limited on high strain level. In order to improve the precision of estimation during high compression, some algorithms have been proposed to expand the 1D window to a $2 \mathrm{D}$ window for avoiding the side-slipping. But they are usually more computationally expensive. In this paper, we proposed a modified 2D multiresolution hybrid method for displacement estimation, which can offer an efficient strain imaging with stable and accurate results. A FEM phantom with a stiffer circular inclusion is simulated for testing the algorithm. The elastographic contrastto-noise rate $(\mathrm{CNRe})$ is calculated for quantitatively comparing the performance of the proposed algorithm with conventional $1 \mathrm{D}$ elastography using phase zero estimation and the 1D elastography using downsampled (d-s) baseband signals. Results show that the proposed method is robust and performs similarly as other algorithms in low strain but is superior when high level strain is applied. Particularly, the CNRe of our algorithm is 15 times higher than original method under $4 \%$ strain level. Furthermore, the execution time of our algorithm is five times faster than other algorithms.
\end{abstract}

\section{Introduction}

Mechanical properties of soft tissue have been used as an important indicator of several diseases, such as breast $[1,2]$, liver, and prostate cancer $[3,4]$. Generally, the carcinoma tissue is stiffer than the surrounding normal tissues. Palpation, as the standard procedure during physical examination, is used to touch and feel tissue below the surface of the body, while its accuracy is limited by the depth of measurement [5]. Ultrasound elastography is a relative new imaging modality for the clinical evaluation of elastic properties of soft tissue using ultrasound. It was first referred to as elastography by Ophir et al. in 1991 [6]. It became a very hot research focus of medical ultrasound in the last two decades $[7,8]$. Major medical imaging device companies have launched their ultrasound products with elastography or shear wave imaging modalities [7-10]. Clinical trials have been conducted for various clinical applications with promising results.

Ultrasound elastography has been developing into an effective method in cancer diagnosis due to its capability and simple implementation [11]. The basic steps of the technique are as follows: (1) the biological tissue is compressed by contact or noncontact way; (2) backscattered radio frequency (RF) signals before and after tissue compression are collected, respectively, by ultrasonic transducer; (3) the tissue displacement estimation algorithm is applied to estimate the displacement field from the RF signals; and (4) the strain field is reconstructed from the displacement field by strain estimation algorithm.

Accurate estimation of tissue displacement is a very important step in ultrasound elastography. Different methods were proposed in the last two decades. Majority of these methods use correlation technique in time domain or the phase domain for displacement estimation [6, 12-14]. In time domain, several matching operators like correlation coefficients, the sum of squared differences (SSD), and the sum of absolute differences (SAD) are employed to finding the optimal matching in the pre- and postcompression RF signals [15, 16]. Since the computational cost of the matching operators in time domain is large, phase-based methods like phase zero 
estimation, which have higher computational efficiency than time-domain methods, have been implemented in clinical ultrasound system for real-time strain imaging [13, 17]. Both $1 \mathrm{D}$ and 2D elastography methods can obtain displacements field, but the $1 \mathrm{D}$ estimation algorithms only consider axial displacements in tissue. They have difficulty in obtaining precise results in complex tissue environment, especially under high compression conditions. In general, the RF data sequence will be shifted laterally because of the lateral displacement in tissue. In order to reduce the errors due to lateral motion in 1D elastography, 2D elastography algorithms like hybrid displacement estimation method and a modified block matching method have been proposed $[18,19]$. These methods consider both axial and lateral displacement; they expand the $1 \mathrm{D}$ window to a $2 \mathrm{D}$ window to avoid the side-slipping of the $1 \mathrm{D}$ window, which can greatly improve the precision of estimation result in high compression situation, but 2D elastography is usually more computational expensive than $1 \mathrm{D}$ elastography.

In this paper, we proposed a modified 2D multiresolution hybrid method for displacement estimation, which can offer an efficient strain imaging with stable and accurate results. To test the algorithm, a heterogeneous computational phantom is simulated using finite element model (FEM), with a rectangle background containing a stiffer circular inclusion. The synthetic RF data are generated from Filed II software [20]. We compare the result with three different algorithms and show a great improvement of our method in ultrasound elastography.

\section{Methods}

In order to make a tradeoff between speed and accuracy, we proposed a method using modified 2D multiresolution hybrid elastography. Preprocessing procedure is first applied to the raw RF data to obtain envelopes and baseline signals at different resolutions. Chen et al. proposed a hybrid displacement estimation method, which applied 3-level estimation based on cross-correlation and weighted phase separation (WPS) [18]. We suggest processing coarse estimation also on 3-level cross-correlation on sampled RF data by different sampling rate. And then a fine estimation is carried out on the whole frame RF date by phase zero-crossing method [15], which uses the results of the coarse estimation as the input to improve the resolution and accuracy.

2.1. Preprocessing. The analytic signals of predeformation and postdeformation radio frequency (RF) signals are obtained by applying Hilbert transformation to the raw RF data. The baseband signals can be calculated by demodulating the analytic signals with a carrier wave $\exp \left(j w_{0} t\right)$, where $w_{0}$ denotes the modulation frequency. The modulation frequency $w_{0}$ should be chosen close to the transducer's center frequency.

The baseband signals are downsampled at different downsample rates. The downsample rates should satisfy the Nyquist sampling condition. The downsampled baseband signals are then converted into amplitude and phase data using FFT. The amplitude data at different scales are used in corresponding coarse to fine estimation, but the phase data are only used for fine estimation.

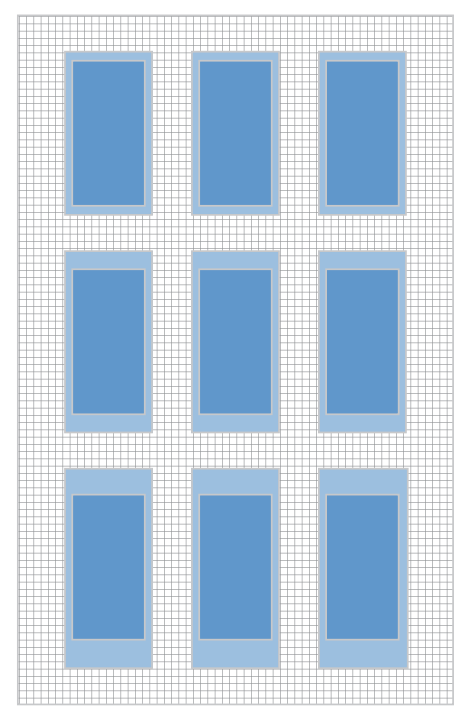

The whole frame

Calculate windows

Search windows

FIGURE 1: Over of the level 1 search, the calculate windows and search windows are equally distributed.

\subsection{Coarse Estimation}

2.2.1. Level 1 Search. Nine evenly distributed windows have been selected in the coarsest scale of the predeformation frame (see Figure 1). Displacements are calculated in these 9 windows by finding the highest correlation coefficient between predeformation and postdeformation frames in a search window $[19,21]$. The correlation coefficient can be expressed as

$$
\begin{aligned}
& R\left(d_{x}, d_{y}\right) \\
& =\frac{\sum_{(x, y) \in T}[A(x, y)-\bar{A}]\left[B\left(x+d_{x}, y+d_{y}\right)-\bar{B}\right]}{\sqrt{\sum_{(x, y) \in T}[A(x, y)-\bar{A}]^{2} \sum_{(x, y) \in T}\left[B\left(x+d_{x}, y+d_{y}\right)-\bar{B}\right]^{2}}},
\end{aligned}
$$

where $d_{x}$ is the lateral displacement, $d_{y}$ is the axial displacement, $A$ and $B$ denote the envelope data from predeformation and postdeformation frame, $\bar{A}$ and $\bar{B}$ are the averages of $A$ and $B$, and $T$ is the window size. Since computational cost at this level is relatively cheap, the size of search windows can be set large enough, and in order to reduce the errors in this level, we do not use multiresolution search method mentioned by Chen in this level. In addition, the size of the search windows in the lower rows is selected bigger than in the upper row since displacement will be accumulated with respect to depth. The output of level 1 search is nine axial-lateral displacement estimations within the nine search windows.

2.2.2. Level 2 Search. Level 2 search is performed at a finer scale than level 1 search. Seven by 11 evenly distributed calculate windows are selected on the predeformation frame. The size of the calculate windows is $1 / 3$ of the size at level 1 


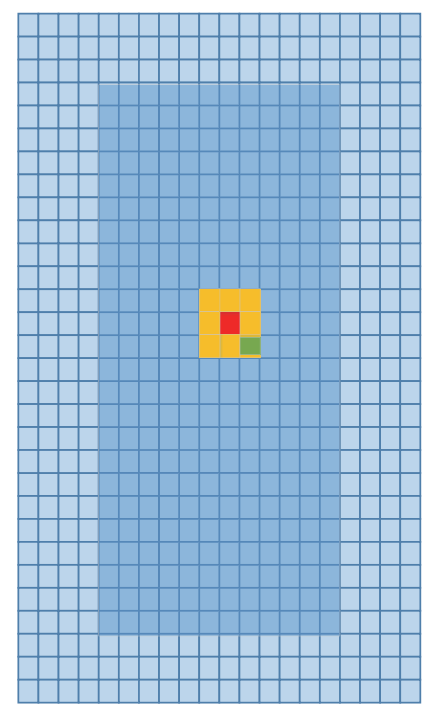

Search window
Target window
Reference point

(a)
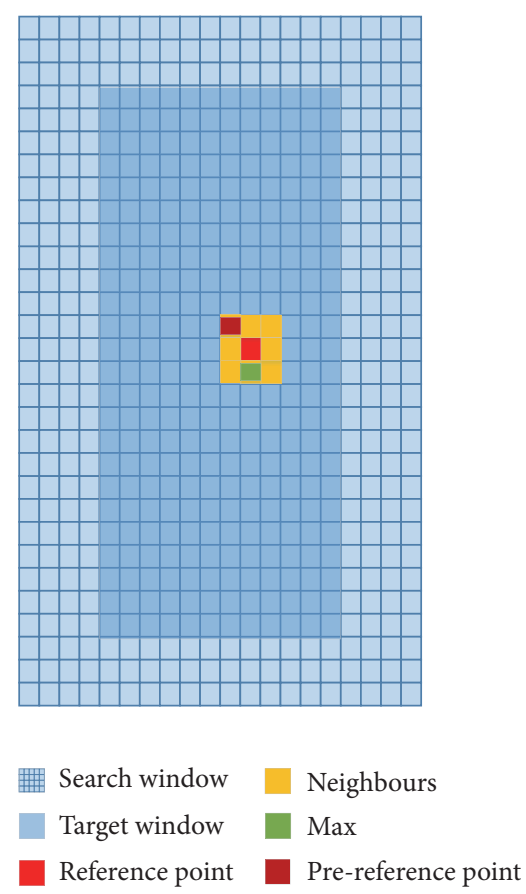

(b)

Figure 2: Level 2 search strategy. (a) Set the initial center point of search window to be a reference point and then find the max in its neighbours according to the correlation coefficient. (b) Propagate the reference and its neighbours and then continue to calculate the correlation coefficient.

and the size of the search windows is bigger than that at level 1 , which is selected according to the deformation degree. The initial axial and lateral displacement estimates of level 2 are inherited from the output of level 1 and bilinear interpolated to the finer scale. The search windows located at the point according to the output and the center of calculate windows.

The so-called "following tracking" strategy (see Figure 2) is used to decide the search direction at each window [22]. First, set the initial center point of search window to be a reference point, and then set the reference point's immediate neighbours to be the search points; after that we need to calculate the correlation coefficient of calculate windows and search windows at each search point. The point which has the highest correlation coefficient will be the next reference point; the search points then propagate to the current reference point's neighbours, and so on. When the reference point occurs at a fixed position or out the range of search window, the calculation in this window is completed, and the axial and lateral displacement estimation is according to the position of the final reference point. Chen et al. used a multiresolution search method in this level; we removed it and increased the numbers of the reference point's neighbours to obtain a more accurate result. We suggested that the increment of computational complexity in this level is meaningful.

However, this time the searches are not independent; there is another delivering strategy that is used to deliver displacement estimations from one window to the next window which on the same column. The next window's initial reference point is no longer the center point of search window, but the position according to the output of the current window.

The advantage of the searching strategy above is that we can find the point which matches the highest correlation coefficient quickly with lower computational complexity. Moreover, the strategy has a good error-correction mechanism to ensure displacement continuity in both axial and lateral direction.

2.2.3. Level 3 Search. The calculate windows and search windows are the smallest in coarse search and this process should finish the whole frame search. The size of calculate windows should be $1 / 2$ of the size at level 2 , and level 3 search stops when the calculate windows cover the whole frame. Similar as in level 2, we also need to interpolate the displacement results in level 2 to level 3 with finer scale. And we use the same search strategy to obtain the axial and lateral displacement in level 3 [22]. Since we do not need further refinement of the lateral displacement, and in order to avoid shifting the signals laterally too much, we extract the significant part of the lateral displacement and only shift the RF signal within that region. The lateral displacement should be smoothed and be considered as the final lateral displacement. There are many kinds of the smooth method; we suggest using the Savitzky-Golay filtering method.

\subsection{Fine Estimation}

2.3.1. Displacement Estimation. The initial axial and lateral displacement estimation over the whole RF data can be 
bilinear interpolated from the coarse estimation from level 3 search. A modified phase zero algorithm is proposed for the fine displacement estimation [13].

Let us denote $x_{1}$ and $x_{2}$ to be the $1 \mathrm{D}$ windowed RF signals before and after compression of the tissue. In elastography, the postdeformation RF data is considered to be a compressed and time-shifted vision of the predeformation signals, and the signal compression can be neglected. Thus, $x_{2}$ can be expressed as

$$
x_{2}(t)=x_{1}(t+\tau) .
$$

In general, the correlation between two signals can be calculated from the cross-correlation function as follows:

$$
\langle a, b\rangle\langle t\rangle=\int_{-\infty}^{\infty} a^{*}\left(t^{\prime}\right) b\left(t^{\prime}+t\right) d t^{\prime} .
$$

Consequently, the signal's autocorrelation function can be a time-shifted modification of the cross-correlation function

$$
\begin{gathered}
\left\langle x_{1}, x_{2}\right\rangle\langle t\rangle=\int_{-\infty}^{\infty} x_{1}^{*}\left(t^{\prime}\right) x_{2}\left(t^{\prime}+t\right) d t^{\prime} \\
=\int_{-\infty}^{\infty} x_{1}^{*}\left(t^{\prime}\right) x_{1}\left(t^{\prime}+t+\tau\right) d t^{\prime}, \\
\int_{-\infty}^{\infty} x_{1}^{*}\left(t^{\prime}\right) x_{1}\left(t^{\prime}+t+\tau\right) d t^{\prime} \\
=\int_{-\infty}^{\infty} x_{1}^{*}\left(t^{\prime}\right) x_{1}\left(t^{\prime}+t+\tau\right) d t^{\prime} \\
=\left\langle x_{1}, x_{1}\right\rangle\langle t+\tau\rangle \\
\left\langle x_{1}, x_{2}\right\rangle\langle t\rangle=\left\langle x_{1}, x_{1}\right\rangle\langle t+\tau\rangle .
\end{gathered}
$$

Since the maximum of the autocorrelation equals the maximum of the cross-correlation, the conventional crosscorrelation determines this maximum to estimate the time shift. When we return the baseband signals to analytic signals, the phase $\varphi(t)$ of the correlation function of the analytic signals $x_{1+}(t)$ and $x_{2+}(t)$ has an identical root

$$
\begin{aligned}
\varphi(-\tau) & =0, \\
\varphi(t) & =\arg \left(\left\langle x_{1+}, x_{2+}\right\rangle(t)\right), \\
x_{1+}(t) & =\exp \left(j \omega_{0} t\right) x_{1}(t), \\
x_{2+}(t) & =\exp \left(j \omega_{0} t\right) x_{2}(t) .
\end{aligned}
$$

Using the Newton iteration, we can figure out the root of $\varphi(t)$ to find the time shift estimation

$$
\begin{aligned}
\tau_{0,0} & =0, \\
\tau_{k, 0} & =\tau_{k-1, N} \\
\tau_{k, l} & =\tau_{k, l-1}-\frac{\varphi\left(t_{n}\right)}{\varphi^{\prime}\left(t_{n}\right)} \approx \tau_{k, l-1}-\frac{\varphi\left(t_{n}\right)}{\omega_{0}} \\
& =\tau_{k, l-1}-\frac{\arg \left(\left\langle x_{1+}, x_{2+}\right\rangle\left(\tau_{k, l-1}\right)\right)}{\omega_{0}} .
\end{aligned}
$$

Then replace it by a sum of oversampled signals

$$
\begin{gathered}
\tau_{k, l}=\tau_{k, l-1}-\frac{1}{w_{0}} \arg \left(\exp \left(j \varpi_{0} \tau_{k, l-1}\right)\right. \\
\left.\quad \sum_{\tau_{k, l-1}-T / 2}^{\tau_{k, l-1}+T / 2} x_{1}^{*}(t) x_{2}\left(t-\tau_{k, l-1}\right)\right),
\end{gathered}
$$

where $\tau_{k, l}$ denotes the displacement estimation in the $k$ th window after iterating for $l$ times and $T$ denotes the calculation window size.

In original phase zero-crossing estimation, the displacement estimation of the previous window will be used as the initial value for the next window [13]. The error of one window will propagate to the remaining windows along the $\mathrm{RF}$ line. This can cause large accumulated errors in the whole frame. On the other hand, this algorithm is based on the assumption that the lateral displacement in the tissue is negligible. However, tissue will be deformed in both the axial and the lateral directions when freehand compression is applied [21]. Lateral displacement should be considered when estimating the whole displacement field.

We propose a modified algorithm to solve the problems described above. We get the coarse estimation including axial and lateral displacement in the previous coarse calculation, so the iteration between the neighbouring windows can be simplified comparing with 1D elastography method. And the lateral deviation will be applied to the computational process. The calculation is independent of each window, so it makes it easier to take this algorithm to be executed based upon CUDA (Compute Unified Device Architecture)

$$
\begin{aligned}
\tau_{0,0} & =0, \\
\tau_{k, 0} & =\tau_{k, \text { coarse }}^{\prime} \\
\tau_{k, l} & =\tau_{k, l-1}-\frac{1}{w_{0}} \arg \left(\exp \left(j \varpi_{0} \tau_{k, l-1}\right)\right. \\
& \left.\cdot \sum_{\tau_{k, l-1}-T / 2}^{\tau_{k,-1}+T / 2} x_{1}^{*}(t) x_{2, \text { shift }}\left(t-\tau_{k, l-1}\right)\right) .
\end{aligned}
$$

The initial value of each window is replaced by the coarse axial displacement $\tau_{k \text {,coarse }}^{\prime}$ on current window. And take the lateral shift on $x_{2}(t)$ to be $x_{2 \text {,shift }}(t)$.

2.3.2. Strain Estimation. Strain is defined as the gradient of the displacement. Here we only calculate the axial strain and the least-squares strain estimator (LSQSE) is used, which employs a piecewise linear curve fitting [23]. After the axial displacement field is obtained, the strain map may be modeled as

$$
u(i)=a z(i)+b,
$$

where $u$ denotes the axial displacement and $z$ is the tissue depth. The constants $a$ and $b$ are the coefficients to be 


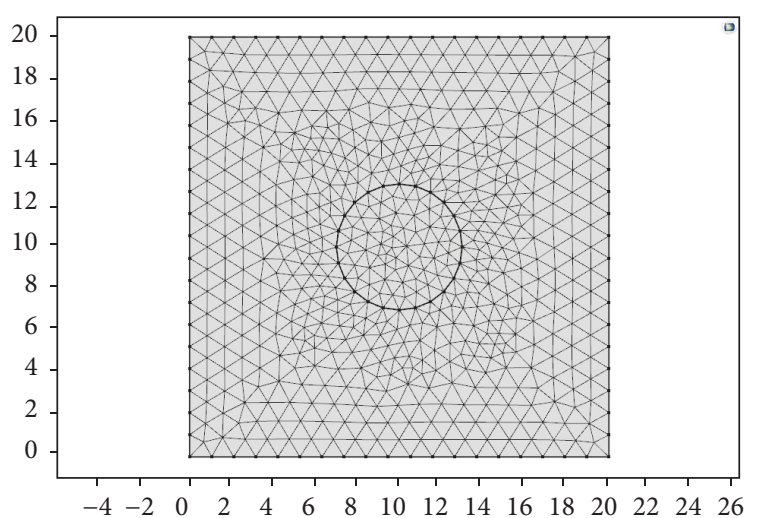

FIGURE 3: Heterogeneous finite element model simulates the stiffer circular inclusion.

estimated, and $a$ represents the local strain. Transform the $z$ to matrix form

$$
\underline{u}=A\left[\begin{array}{l}
a \\
b
\end{array}\right]=\left[\begin{array}{cc}
z(1) & 1 \\
z(2) & 1 \\
\cdots & 1 \\
\cdots & 1 \\
z(N) & 1
\end{array}\right] \cdot\left[\begin{array}{l}
a \\
b
\end{array}\right] .
$$

The least square solution is given by

$$
\left[\begin{array}{l}
\widehat{a} \\
\widehat{b}
\end{array}\right]=A^{T}\left[A A^{T}\right]^{-1} \widehat{u},
$$

where $\widehat{a}$ and $\widehat{b}$ denote the estimation of $a$ and $b$, respectively.

2.4. Simulation. A FEM (finite element model) phantom is simulated using Commercial FEM software COMSOL Multiphysics 5.0 (COMSOL USA). FIELD II software is used for ultrasound simulations. The size of rectangle background of the $2 \mathrm{D}$ model is $20 \mathrm{~mm} \times 20 \mathrm{~mm}$, with a stiffer circular inclusion in the center. The radius of the inclusion is $3 \mathrm{~mm}$. Triangular mesh was generated and refined automatically by COMSOL (see Figure 3). Smooth displacement and strain field can be obtained for further RF signal simulation. The inclusion and its background have same density of $1000 \mathrm{~kg} / \mathrm{m} 3$ and Poisson's ratio of 0.495 . Young's modulus of the inclusion is $100 \mathrm{kPa}$, which is multiple times stiffer than the background. In this study, we set strain contrast between lesion and background to be 5 , so that there will be a clear boundary and CNRe can be easily used for performance comparison. The phantom is compressed by uniaxial compression with axial displacement set to $0.3 \mathrm{~mm}-0.8 \mathrm{~mm}$ (i.e., the axial strain is $1.5 \%-4.0 \%$ ). The bottom of the phantom is fixed.

A 192-element linear array transducer (64 active elements) with a center frequency of $7.5 \mathrm{Mhz}$ is simulated. The transducer has a pitch of $0.255 \mathrm{~mm}$ and an element height of $5 \mathrm{~mm}$. The element width is equal to the wavelength. The number of scan-lines is 128 and the distance between adjacent lines is equal to the pitch. The transmitting focus is at $30 \mathrm{~mm}$

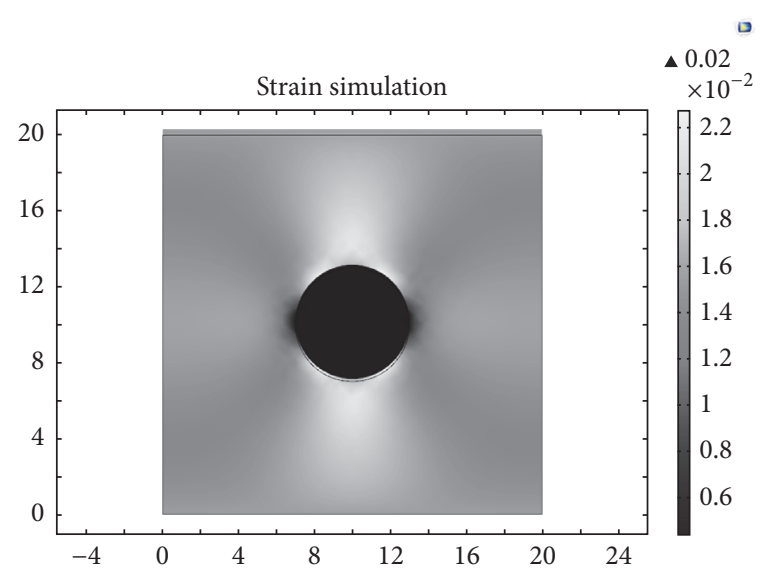

FIgURE 4: Strain map simulated by COMSOL as the ground truth.

and dynamic focusing with focal zones step by $1 \mathrm{~mm}$ is used for receiving focus. The image zone has a width of $20 \mathrm{~mm}$ and a depth of $20 \mathrm{~mm}$. The speed of sound is assumed to be $1540 \mathrm{~m} / \mathrm{s}$ and the sampling frequency of the RF signals is $120 \mathrm{Mhz}$. The original scatters are randomly distributed in the image zone with random scattering amplitude. The predeformation RF signals are simulated with FIELD II using the parameters described above $[20,24]$. The new scatters positions after compression are calculated according to COMSOL simulation. The postdeformation RF signals are then simulated with FIELD II using the new scatterer distribution.

\section{Results and Discussion}

Ultrasound RF data was generated using computer simulation. With the synthetic RF data, the displacement and strain distribution were calculated using the modified 2D multiresolution hybrid elastography. Comparison was made between the proposed method, the original $1 \mathrm{D}$ elastography and the 1D elastography using downsampled (d-s) baseband signals. Since the modified method has a downsampled step, we make a downsampled version of $1 \mathrm{D}$ elastography to compare with the proposed method. To achieve that, we use the same processes as the $1 \mathrm{D}$ elastography method to get envelop signals, and the envelop signals will be then downsampled and further $1 \mathrm{D}$ elastography calculations will be performed on the downsampled envelop signals.

Figure 4 shows the theoretical strain maps using finite element analysis in COMSOL as the ground truth. Figure 5 presents the estimated strains obtained by three algorithms for the computational phantom under different applied strain. The direct viewing of the result shows that $1 \mathrm{D}$ elastography is slightly better under low strain level; the reason is that the $1 \mathrm{D}$ method build on the whole RF data and the iterative calculation is more complete and precise under low strain level. The performance of the algorithm we proposed is similar to $1 \mathrm{D}$ algorithm when low strain is applied but much better under higher strain level. As we can see in the first two columns, some error appeared in the center of the 


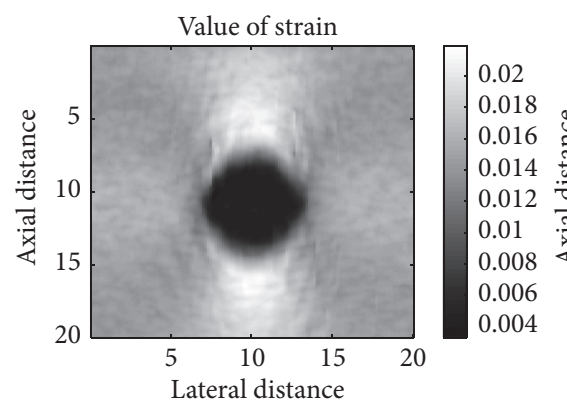

(a)

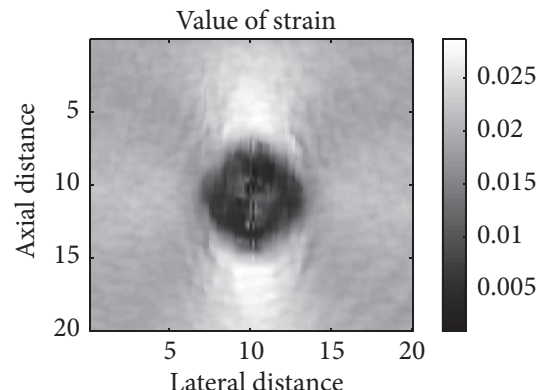

(b)

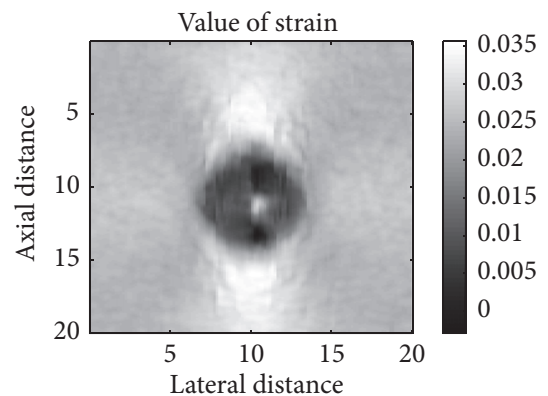

(c)

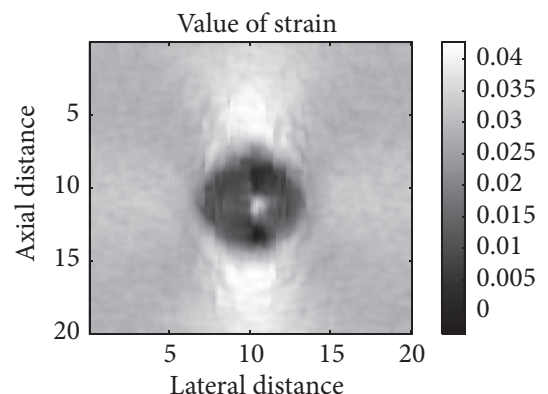

(d)

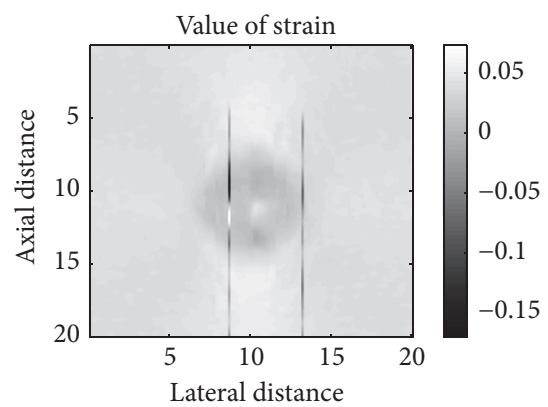

(e)

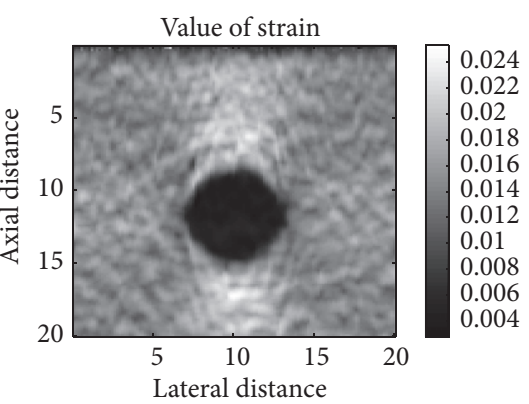

(f)

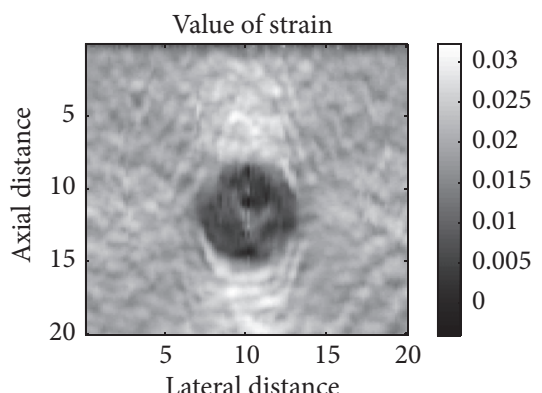

(g)

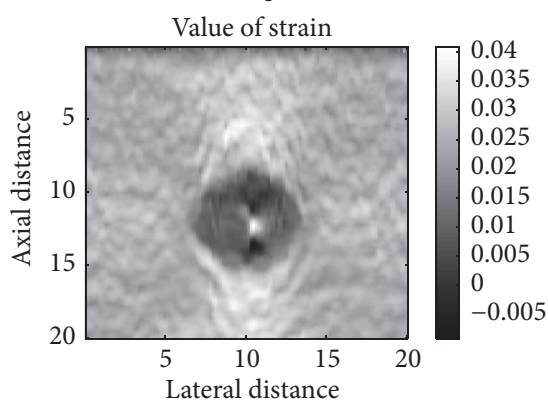

(h)

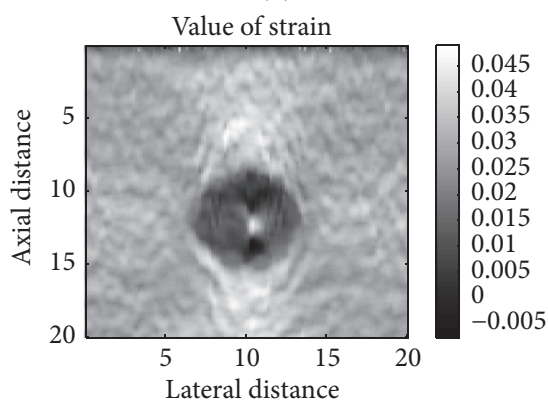

(i)

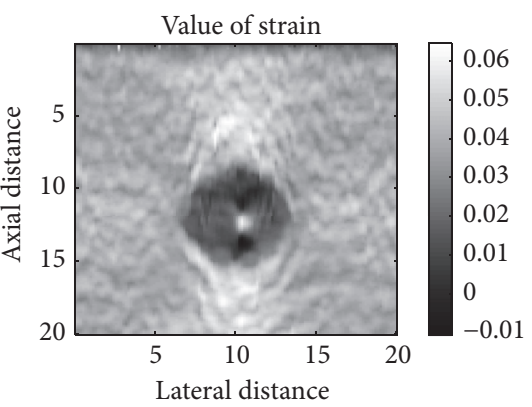

(j)

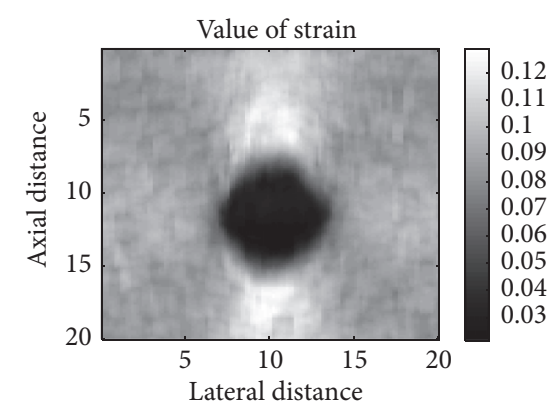

(k)

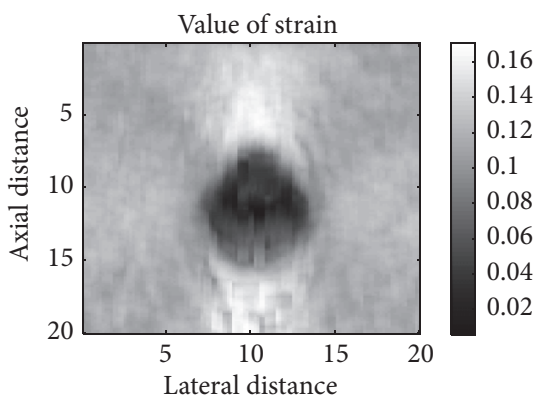

(l)

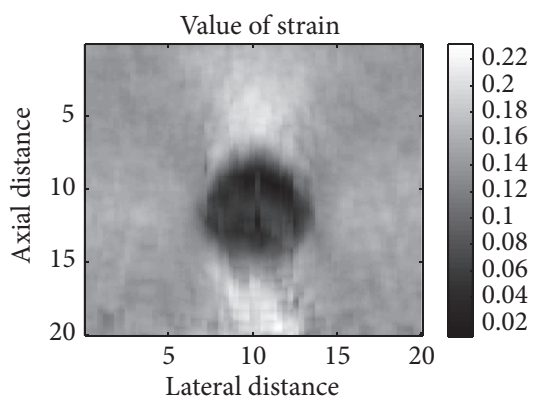

(m)

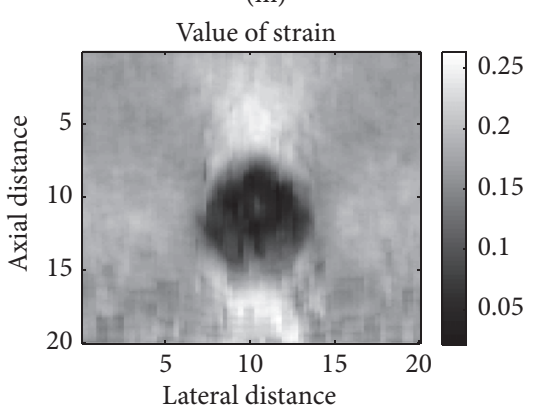

(n)

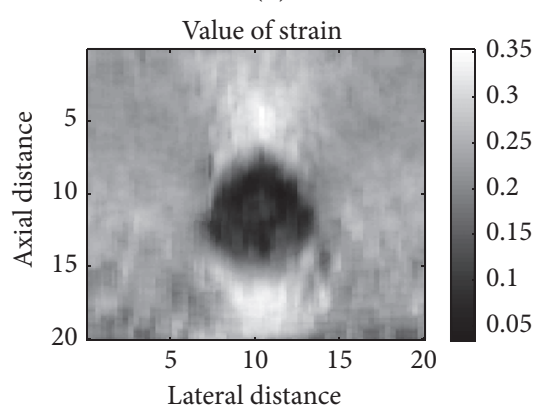

(o)

Figure 5: Strain maps of three different algorithms. Different strain levels as 1.5\%, 2.0\%, 3.0\%, 3.5\%, and 4.0\% are applied at each row. The first column ((a)-(e)), the second column ((f)-(j)), and the third column ((k)-(o)) show, respectively, the strain map obtained by original 1D elastography and original 1D elastography with downsampled and modified 2D multiresolution hybrid elastography. 


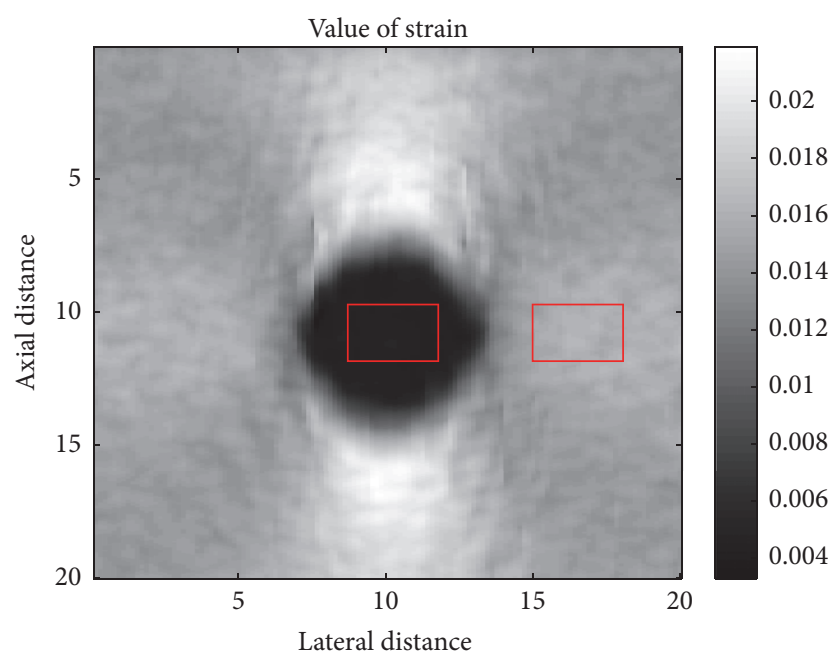

Figure 6: The extra area of inclusion and background for the calculation of CNRe and the average estimated strain rate.

result when strain level increased, and there are two error lines in 1D elastography (Figure 5(e)) because of the lacking consideration of lateral displacement and iterative calculation between two windows.

To quantify the performance of different algorithms, CNRe is calculated at each strain level and we use the binary strain image to measure the accuracy of each algorithm [25]. The definition of the CNRe is provided in the following:

$$
\mathrm{CNR}_{\mathrm{e}}=\frac{2\left(s_{1}-s_{2}\right)^{2}}{\sigma_{s_{1}}^{2}+\sigma_{s_{2}}^{2}},
$$

where $s_{1}$ and $\sigma_{s_{1}}^{2}$ denote the mean and the variance of strain estimation in the inclusion and $s_{2}$ and $\sigma_{s_{2}}^{2}$ are the mean and the variance of strain estimation in the background (see Figure 6). The larger the CNRe value, the better the clarity of the results.

We have made a record of the average estimated strain rate for both inclusion and background for comparison purpose. The ratio of $s_{1}$ and $s_{2}$ previously mentioned is shown directly in Figure 7. We can see that the three different algorithms have shown similar average strain ratio between lesion and background in different strain level.

We make a complete test with three algorithms under different strain level range $0.2 \%$ to $4 \%$. Same as $1 \mathrm{D}$ algorithms, the CNRe of our algorithms is too low to distinguish between the lesion and background when strain level is lower than $0.8 \%$, which means the noise on strain estimation will blur the boundary between lesion and background. And the CNRe of the modified 2D multiresolution hybrid elastography algorithm is much higher than the other two algorithms at high strain level (see Figure 8). We can see that the original 1D elastography has a slight better result in low strain level; the reason is that the $1 \mathrm{D}$ method build on the whole RF data and the iterative calculation is more complete and precise under low strain level. The performance of the algorithm we proposed is similar to $1 \mathrm{D}$ algorithm when low strain is applied but better under higher strain level.

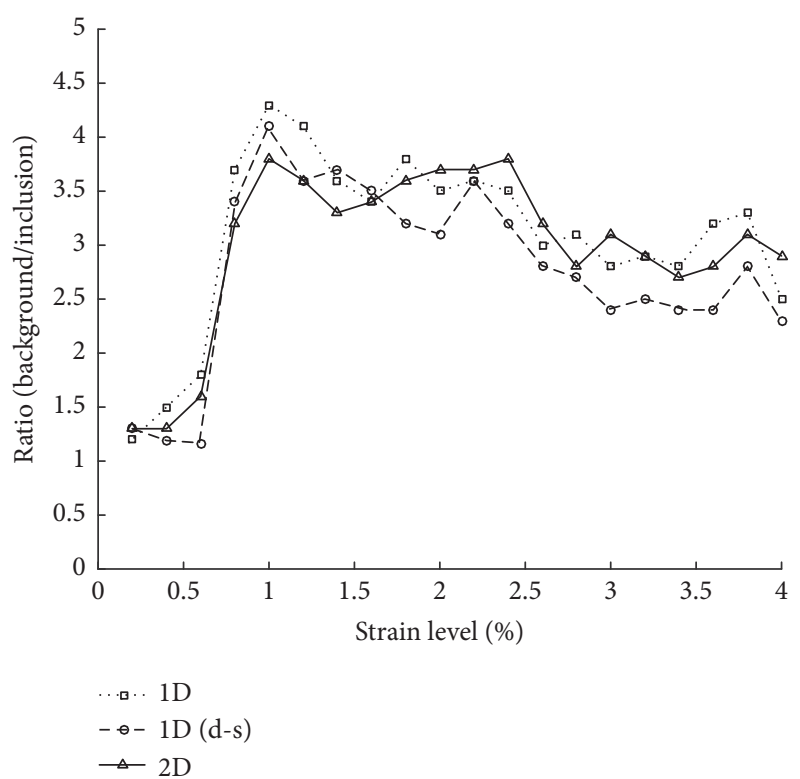

FIGURE 7: The ratio of average estimated strain rates of the inclusion and the background.

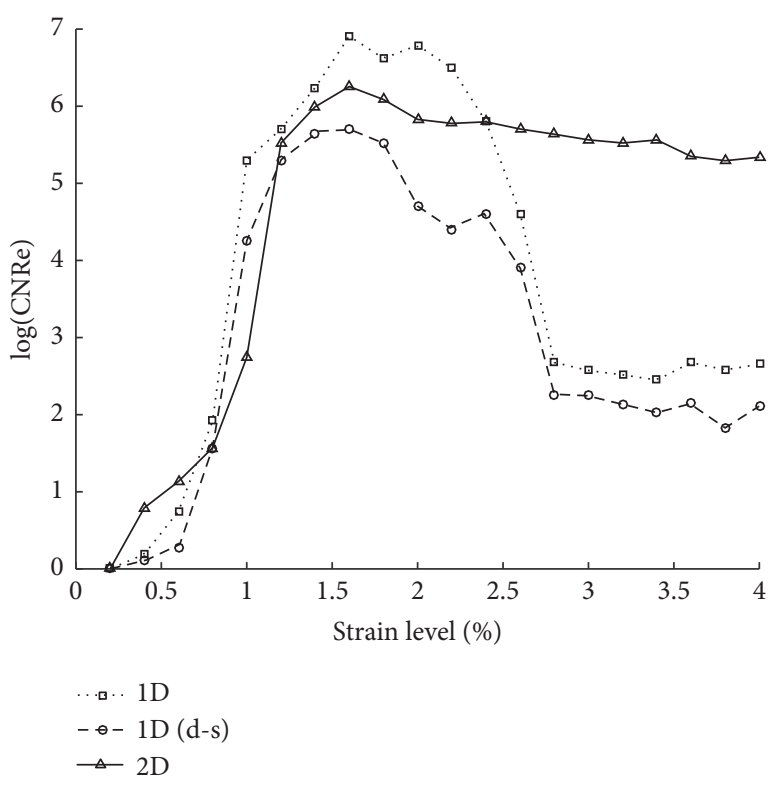

FiguRE 8: The CNRe results of three different algorithms.

The strain binary images (see Figure 9) are obtained by thresholding the strain map. We set the threshold at the same level and plot the binary images, respectively. It is an immediate way to see the accuracy of each strain image. We set the average estimated strain rate for whole phantom as the threshold of the binary images. Additionally, the inclusion area ratios are calculated and compared with the ground truth of FEA result. Then we calculate the inclusion area ratio as the numbers of black pixels and all pixels in the image. The standard denotes the inclusion area ratio in FEA model and results of calculated strain ratio from three methods 


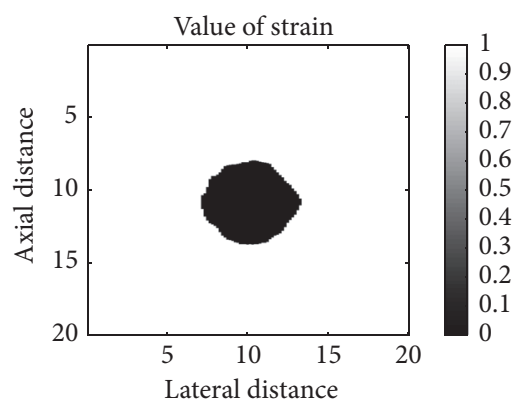

(a)

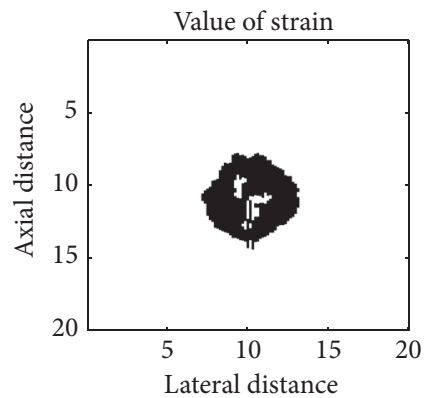

(b)

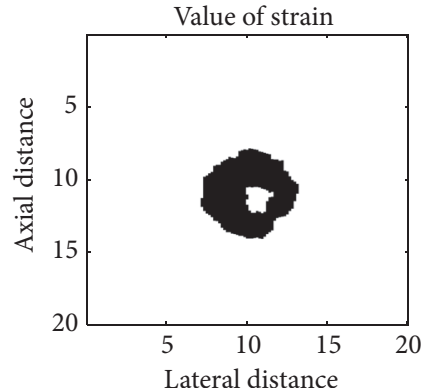

(c)

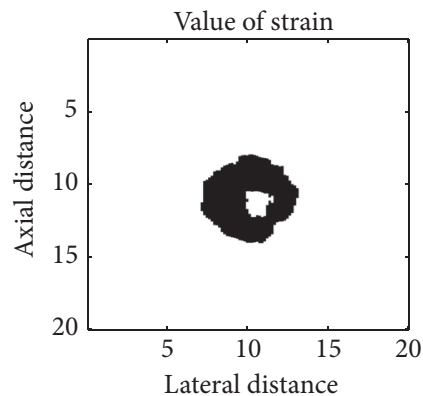

(d)

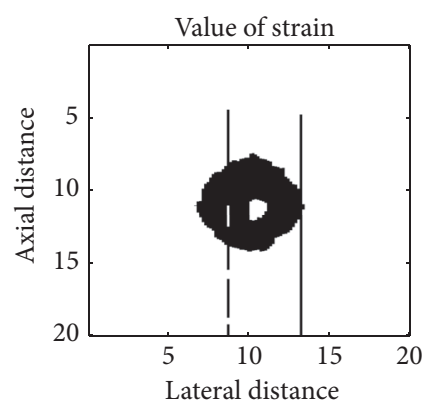

(e)

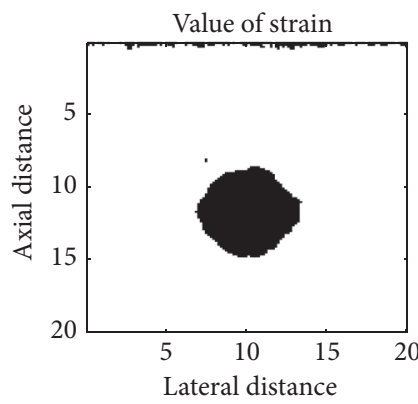

(f)
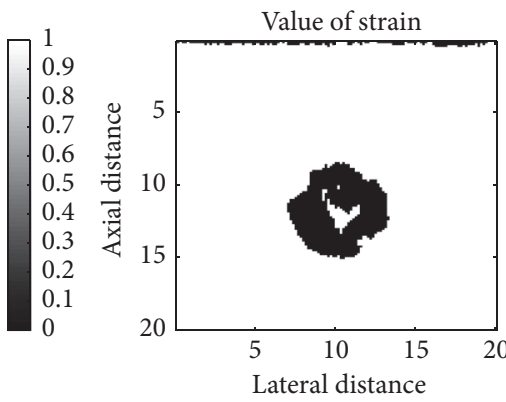

(g)
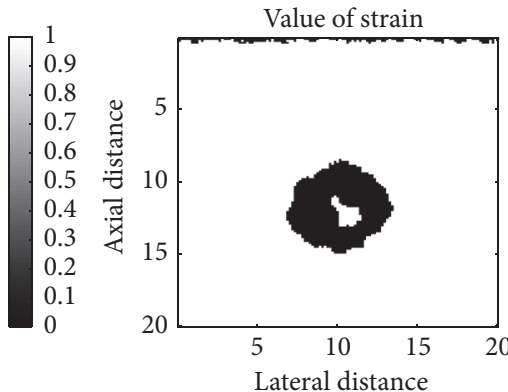

(h)
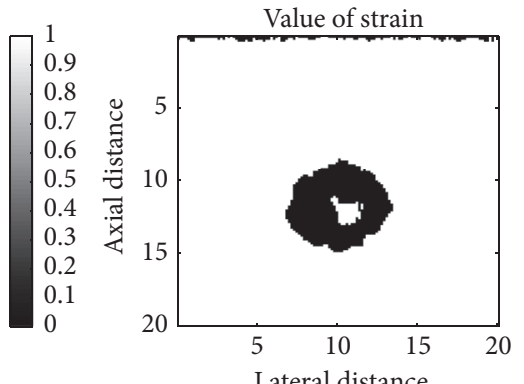

(i)
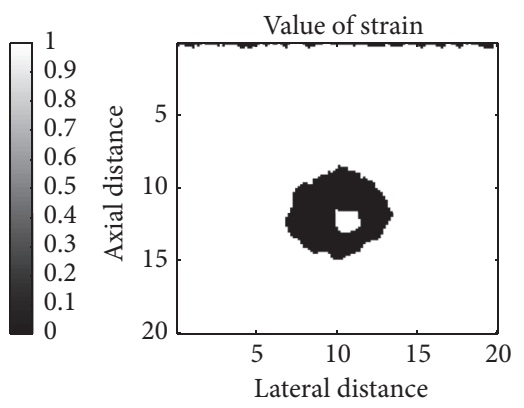

(j)

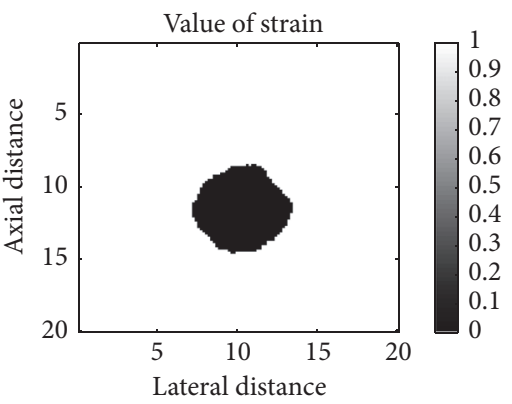

(k)

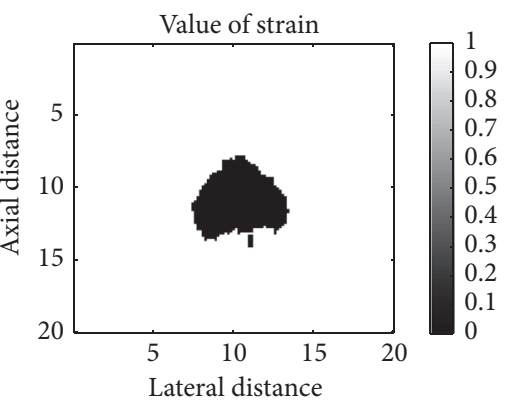

(1)

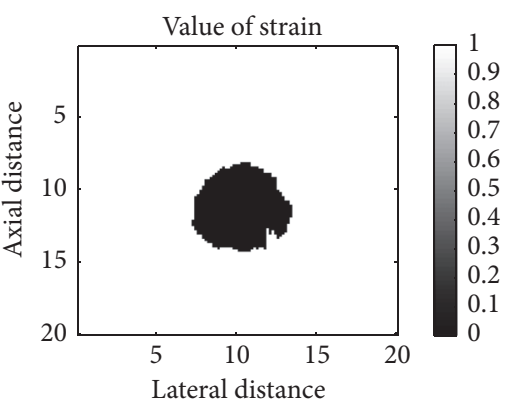

(m)

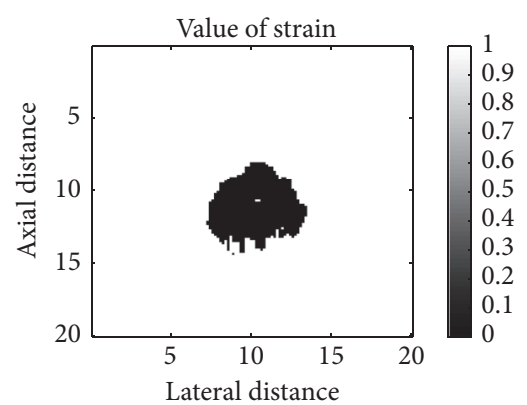

(n)

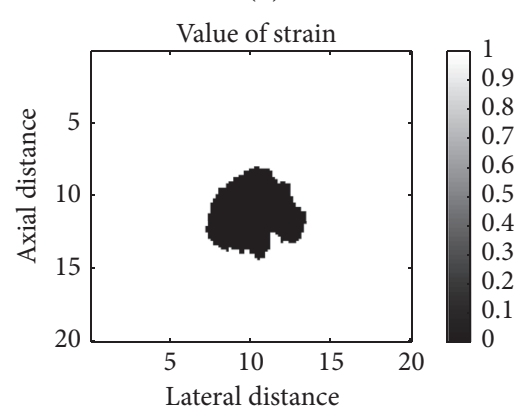

(o)

Figure 9: Strain binary images of three different algorithms. Different strain levels as $1.5 \%, 2.0 \%, 3.0 \%, 3.5 \%$, and $4.0 \%$ are applied at each column. The first column ((a)-(e)), the second column ((f)-(j)), and the third column $((\mathrm{k})-(\mathrm{o}))$ show, respectively, the strain binary map obtained by the corresponding strain map using $1 \mathrm{D}$ elastography, downsampled $1 \mathrm{D}$ elastography, and the proposed 2D elastography method. 


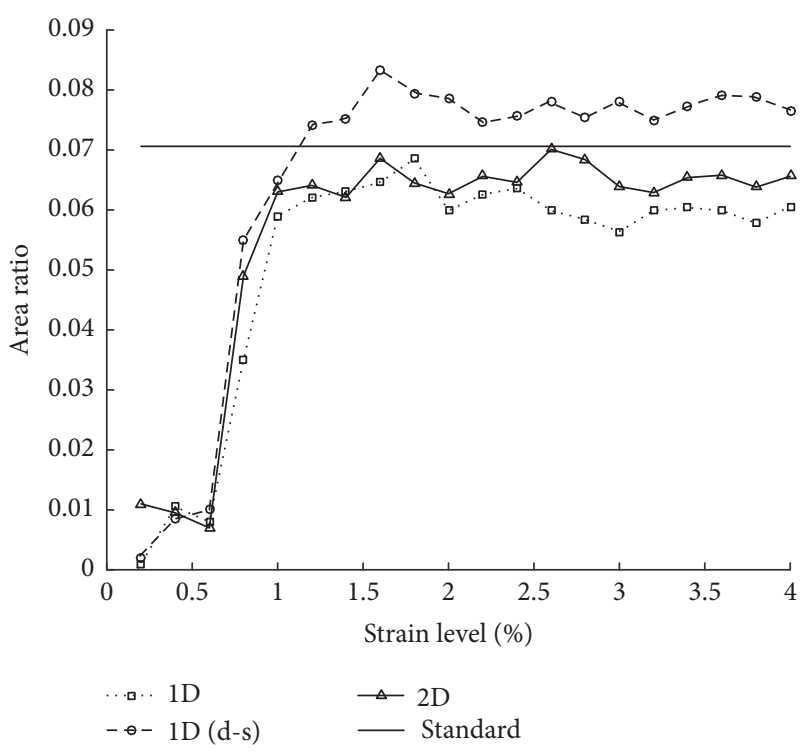

FIGURE 10: The inclusion area ratios results of three different algorithms, where the standard denotes the inclusion area ratio in FEA model.

TABLE 1: Execution times of three different algorithms.

\begin{tabular}{lccc}
\hline Method & 1D & 1D with downsampled & Modified 2D \\
Times (s) & 10.2 & 2.3 & 1.8 \\
\hline
\end{tabular}

are shown in Figure 10. The inclusion area ratio of the new method is closer to the standard than other methods.

In the algorithm we proposed, the lateral resolution depends on the amount of the windows in level 3 search. The lateral resolution increased with the increasing of the amount until the resolution is equal to the sampling frequency of the $\mathrm{RF}$ data. The axial resolution is determined by the physical size of transducer such as the kerf of the array element. The purpose of the consideration of lateral displacement in our algorithm is not to improve the later resolution but to compensate for the interference of lateral offset and get higher accuracy in axial strain image.

All the algorithms were executed with an Intel(R) Core(TM) i7-4790K CPU @2.40 GHz 8.00GB RAM, and MATLAB 2014b was used for implementing and testing them on Windows operation system. The execution times of the different methods are shown in Table 1. The modified 2D multiresolution hybrid elastography algorithm has a shorter time than the other algorithms.

\section{Conclusion}

A modified 2D multiresolution hybrid method has been proposed in this paper. Using finite element model phantom under different strain levels, we have shown that the new algorithm can achieve better CNRe comparing with different methods including original $1 \mathrm{D}$ elastography method and original 1D elastography method with downsampled (d-s). These results demonstrate that the method we suggested is robust and accurate when high level strain is applied. The result of execution time has shown that the new framework has a higher efficiency that it may well be more suitable for real-time application in clinical practice. The limitation of our algorithm is that, to a great extent, the accuracy of the final result is determined by the output of coarse estimation. The strain image calculated by our algorithm is slightly worse than original $1 \mathrm{D}$ elastography under low strain level. In this study, we use simulated data to compare the performance of the proposed method with other methods in different strain level, since ground truth of strain map can be easily obtained. In our next study, we will test our method using phantom and in vivo data.

\section{Conflicts of Interest}

The authors declare that there are no conflicts of interest regarding the publication of this paper.

\section{Acknowledgments}

This work is supported by the National Key Technology Research and Development Program of the Ministry of Science and Technology of China (Grant no. 2017YFC0107202), the Funds for Technology of Suzhou, China (Grants nos. SYG201505 and SZS201510), the Funds for Jiangsu Provincial Key Research and Development Plan (Grant no. BE2017601), and International Cooperation Program of Jiangsu Province (Grants nos. BZ2016023 and BK20161235).

\section{References}

[1] B. S. Garra, E. I. Cespedes, J. Ophir et al., "Elastography of breast lesions: initial clinical results," Radiology, vol. 202, no. 1, pp. 7986, 1997.

[2] A. Itoh, "Review of the techniques and diagnostic criteria of breast ultrasound elastography," Medix Hitachi Supplement, vol. 8, 2007.

[3] M. Friedrich-Rust, M. F. Ong, E. Herrmann et al., "Real-time elastography for noninvasive assessment of liver fibrosis in chronic viral hepatitis," American Journal of Roentgenology, vol. 188, no. 3, pp. 758-764, 2007.

[4] K. König, U. Scheipers, A. Pesavento, A. Lorenz, H. Ermert, and T. Senge, "Initial experiences with real-time elastography guided biopsies of the prostate," The Journal of Urology, vol. 174, no. 1, pp. 115-117, 2005.

[5] J.-L. Gennisson, T. Deffieux, M. Fink, and M. Tanter, "Ultrasound elastography: Principles and techniques," Diagnostic and Interventional Imaging, vol. 94, no. 5, pp. 487-495, 2013.

[6] J. Ophir, I. Céspedes, H. Ponnekanti, Y. Yazdi, and X. Li, "Elastography: a quantitative method for imaging the elasticity of biological tissues," Ultrasonic Imaging, vol. 13, no. 2, pp. 111134, 1991.

[7] J. Ophir, S. Srinivasan, R. Righetti, and A. Thittai, "Elastography: A decade of progress (2000-2010)," Current Medical Imaging Reviews, vol. 7, no. 4, pp. 292-312, 2011.

[8] K. J. Parker, M. M. Doyley, and D. J. Rubens, "Imaging the elastic properties of tissue: the 20 year perspective.," Physics in Medicine and Biology, vol. 56, no. 1, p. -R29, 2011. 
[9] J. Ophir, S. K. Alam, B. S. Garra et al., "Elastography: Imaging the elastic properties of soft tissues with ultrasound," Journal of Medical Ultrasonics, vol. 29, no. WINTER, pp. 155-171, 2002.

[10] A. P. Sarvazyan, O. V. Rudenko, S. D. Swanson, J. B. Fowlkes, and S. Y. Emelianov, "Shear wave elasticity imaging: a new ultrasonic technology of medical diagnostics," Ultrasound in Medicine \& Biology, vol. 24, no. 9, pp. 1419-1435, 1998.

[11] M. M. Doyley, "Model-based elastography: a survey of approaches to the inverse elasticity problem," Physics in Medicine and Biology, vol. 57, no. 3, pp. R35-R73, 2012.

[12] R. Zahiri-Azar and S. E. Salcudean, "Motion estimation in ultrasound images using time domain cross correlation with prior estimates," IEEE Transactions on Biomedical Engineering, vol. 53, no. 10, pp. 1990-2000, 2006.

[13] A. Pesavento, "A time-efficient and accurate strain estimation concept for ultrasonic elastography using iterative phase zero estimation," IEEE Transactions on Ultrasonics, Ferroelectrics and Frequency Control, vol. 46, no. 5, pp. 1057-1067, 1999.

[14] M. O'Donnell, A. Skovorada, and B. Shapo, "Measurement of arterial wall motion using Fourier based speckle tracking algorithms," in Proceedings of the IEEE 1991 Ultrasonics Symposium, pp. 1101-1104, Orlando, Fla, USA.

[15] F. Viola and W. F. Walker, "A comparison of the performance of time-delay estimators in medical ultrasound," IEEE Transactions on Ultrasonics, Ferroelectrics and Frequency Control, vol. 50, no. 4, pp. 392-401, 2003.

[16] S. Langeland, J. D’Hooge, H. Torp, B. Bijnens, and P. Suetens, "Comparison of time-domain displacement estimators for twodimensional RF tracking," Ultrasound in Medicine \& Biology, vol. 29, no. 8, pp. 1177-1186, 2003.

[17] J. E. Lindop, G. M. Treece, A. H. Gee, and R. W. Prager, "Phasebased ultrasonic deformation estimation," IEEE Transactions on Ultrasonics, Ferroelectrics and Frequency Control, vol. 55, no. 1, pp. 94-111, 2008.

[18] L. Chen, R. J. Housden, G. M. Treece, A. H. Gee, and R. W. Prager, "A hybrid displacement estimation method for ultrasonic elasticity imaging," IEEE Transactions on Ultrasonics, Ferroelectrics and Frequency Control, vol. 57, no. 4, pp. 866-882, 2010.

[19] Y. Zhu and T. J. Hall, "A modified block matching method for real-time freehand strain imaging," Ultrasonic Imaging, vol. 24, no. 3, pp. 161-176, 2002.

[20] A. Jensen J and Field., "A program for simulating ultrasound systems," in Proceedings of the 10th Nordicbaltic Conference on Biomedical Imaging, vol. 4, Supplement 1, pp. 351-353, 1996.

[21] H. Shi and T. Varghese, "Two-dimensional multi-level strain estimation for discontinuous tissue," Physics in Medicine and Biology, vol. 52, no. 2, article no. 006, pp. 389-401, 2007.

[22] L. Chen, G. M. Treece, J. E. Lindop, A. H. Gee, and R. W. Prager, "A quality-guided displacement tracking algorithm for ultrasonic elasticity imaging," Medical Image Analysis, vol. 13, no. 2, pp. 286-296, 2009.

[23] F. Kallel and J. Ophir, "A least-squares strain estimator for elastography," Ultrasonic Imaging, vol. 19, no. 3, pp. 195-208, 1997.

[24] J. A. Jensen and P. Munk, "Computer phantoms for simulating ultrasound B-Mode and CFM Images," in Acoustical Imaging, vol. 23, pp. 75-80, 1997.

[25] T. Varghese and J. Ophir, "An analysis of elastographic contrastto-noise ratio," Ultrasound in Medicine \& Biology, vol. 24, no. 6, pp. 915-924, 1998. 


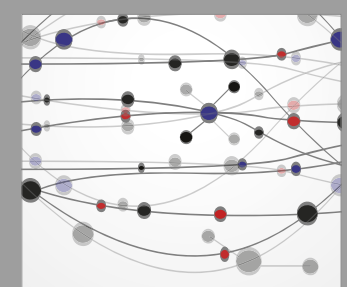

The Scientific World Journal
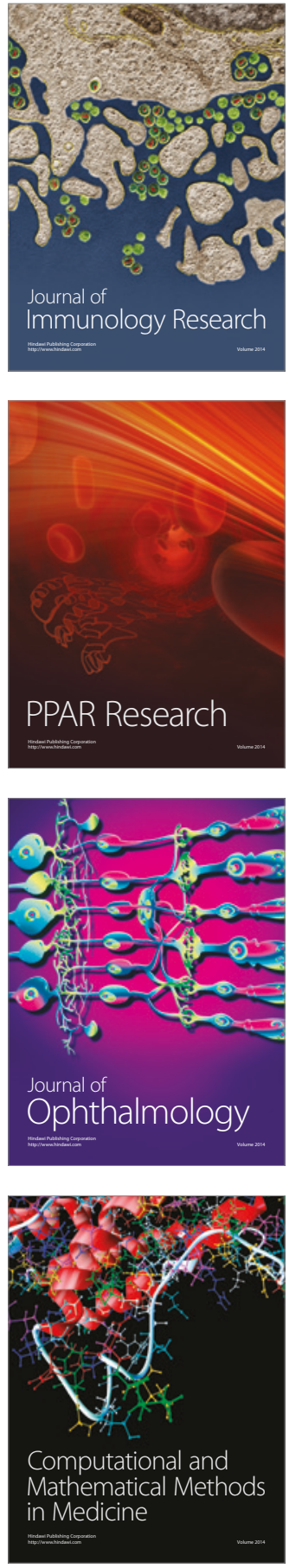

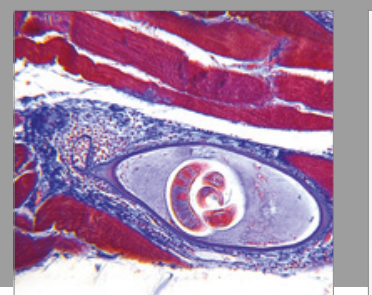

Gastroenterology Research and Practice
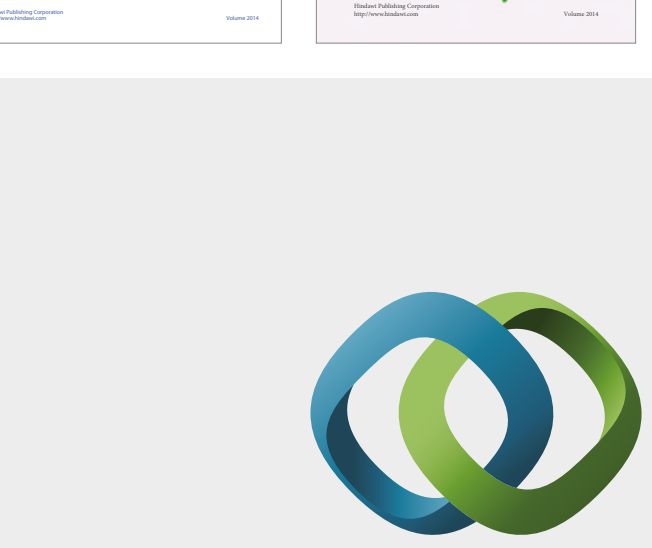

\section{Hindawi}

Submit your manuscripts at

https://www.hindawi.com
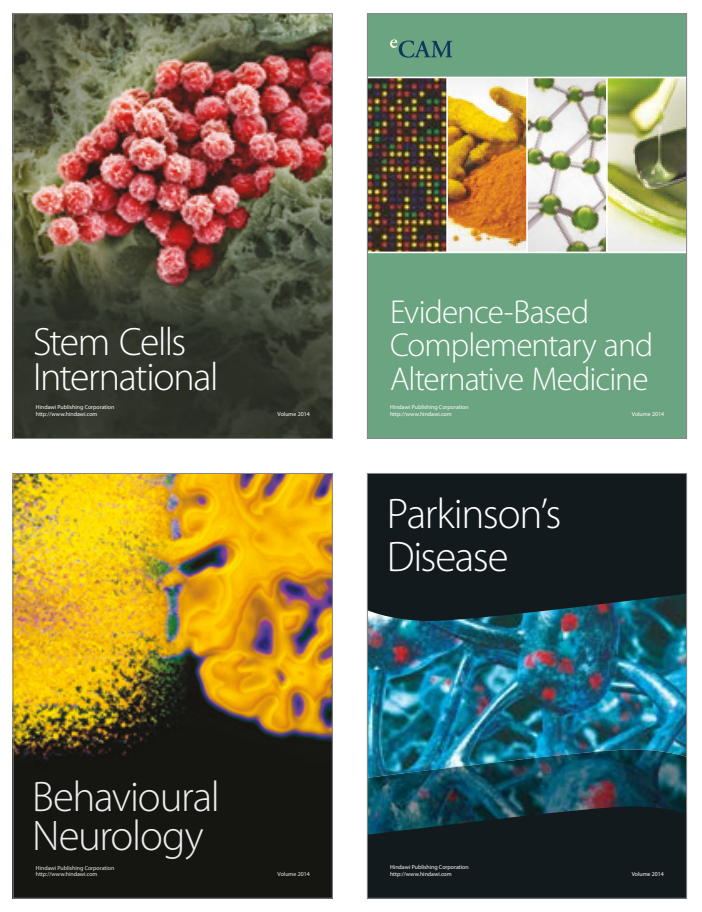
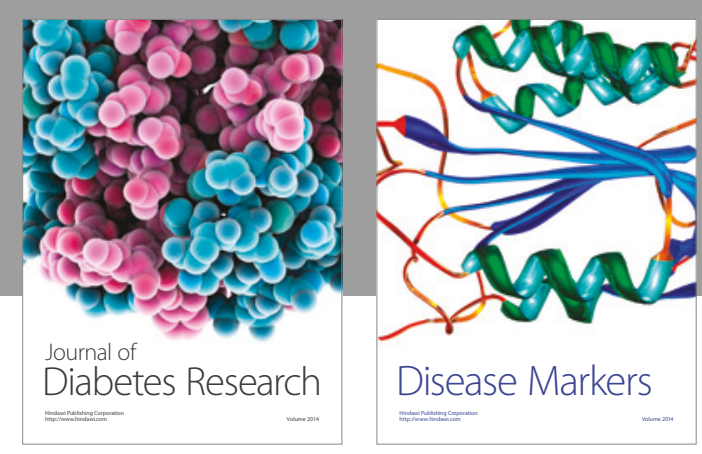

Disease Markers
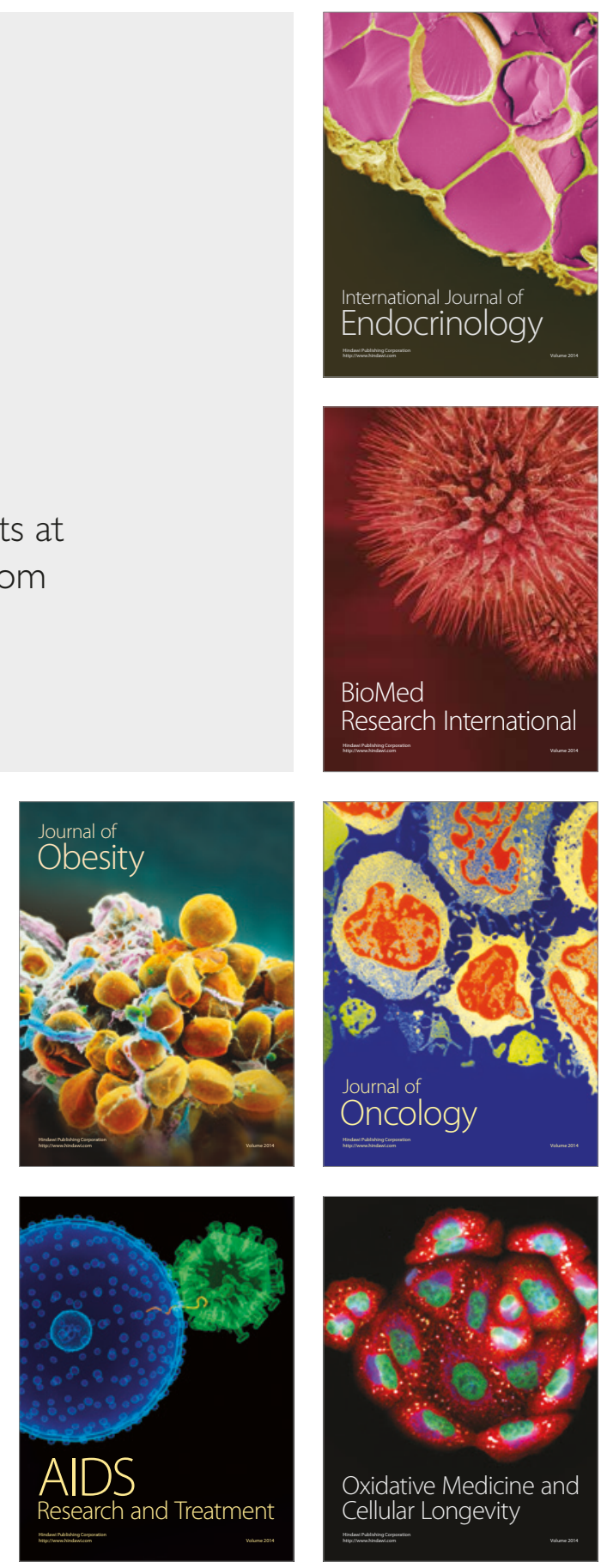